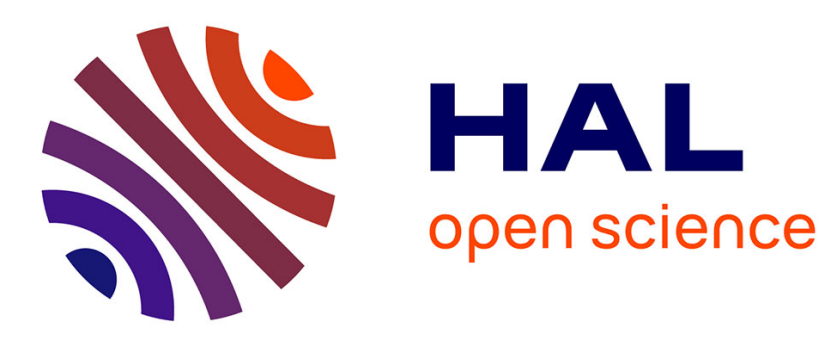

\title{
Observatoire des sciences de l'univers de Grenoble Rapport Hcéres
}

\section{To cite this version:}

Rapport d'évaluation d'une entité de recherche. Observatoire des sciences de l'univers de Grenoble. 2010, Université Joseph Fourier - Grenoble - UJF, Grenoble INP, Université Savoie Mont Blanc, Laboratoire central des ponts et chaussées, Institut de recherche pour le développement - IRD. hceres02032420

\section{HAL Id: hceres-02032420 \\ https://hal-hceres.archives-ouvertes.fr/hceres-02032420}

Submitted on 20 Feb 2019

HAL is a multi-disciplinary open access archive for the deposit and dissemination of scientific research documents, whether they are published or not. The documents may come from teaching and research institutions in France or abroad, or from public or private research centers.
L'archive ouverte pluridisciplinaire HAL, est destinée au dépôt et à la diffusion de documents scientifiques de niveau recherche, publiés ou non, émanant des établissements d'enseignement et de recherche français ou étrangers, des laboratoires publics ou privés. 


\section{a ${ }^{* *}$ res}

agence d'évaluation de la recherche et de l'enseignement supérieur

\section{Section des Unités de recherche}

Rapport de l'AERES sur la structure fédérative :

Observatoire des Sciences de l'Univers de Grenoble (OSUG)

sous tutelle des établissements et organismes:

Université de Grenoble 1 - Joseph Fourier CNRS / INSU IRD INP 


\section{$a^{* *}$ res}

agence d'évaluation de la recherche et de l'enseignement supérieur

Rapport de l'AERES sur la struc ture fédérative :

Observatoire des Sciences de l'Univers de Grenoble (OSUG)

Sous tutelle des éta blissements et organismes

Université de Grenoble 1 - J oseph Fourier

CNRS / INSU

\section{IRD}

INP
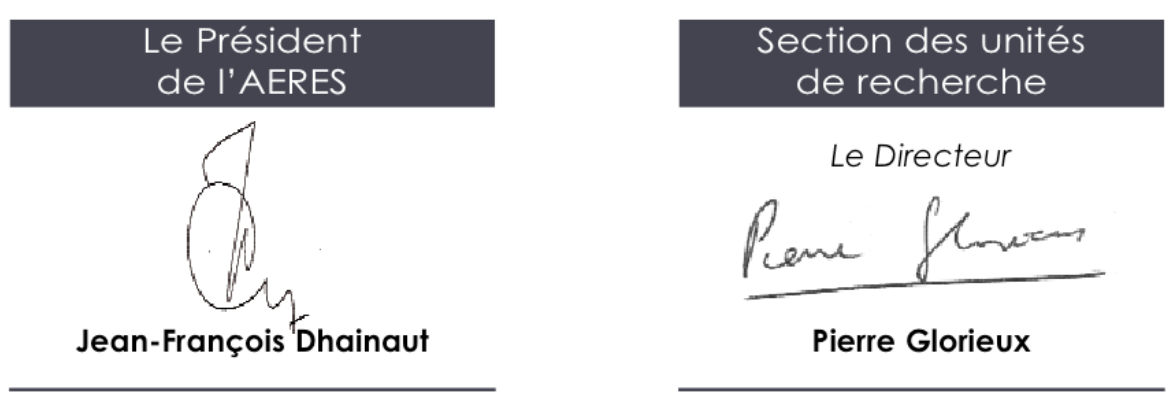

Mai 2010 


\section{Fédération}

Nom de la fédération : osug

Label demandé :

$N^{\circ}$ si renouvellement :

Nom du directeur : M. Henri-Claude NATAF

\section{Membres du comité d'experts}

Président :

M. Michel BLANC, Ecole Polytechnique, Palaiseau

Experts :

M. Yves BARBIN, LSEET, Toulon

M. Regis COURTIN, Observatoire de Paris

M. Philippe DAVY, OSUR, Rennes

M. Yves GAUDEMER, IPGP, Paris

M. Michel PERAULT, ENS, Paris

M. Serge PLANTON, CNRM-GAME, Toulouse

M. Daniel VIDAL MADJAR, UVSQ, Versailles-St-Quentin

\section{Représentants présents lors de la visite}

Délégué scientifique représentant de l'AERES :

M. Daniel GUEDALIA

Représentant(s) des établissements et organismes tutelles:

M. Farid OUABDESSELAM, Président UJF

M. François RENARD, VP adjoint UJF

M. Pierre SOLER, IRD

M. Didier GEORGES, VP recherche INPG

M. Eric BARTHELEMY, INP

M. Dominique LE QUEAU, Directeur INSU, par vidéo- conférence

M. André MARIOTTI, DAS INSU, par vidéo- conférence

M. Patrick MONFRAY, DAS INSU, par vidéo- conférence

Mme Pascale BUKHARI, Déléguée régionale CNRS 


\section{Rapport}

\section{Introduction}

\section{- Déroulement de l'évaluation :}

La visite s'est déroulée sur trois demi-journées, les 7 et 8 février 2010. Une première partie a été consacrée à des exposés par la direction de l'OSUG sur sa politique générale, les volets recherche, formation et Services d'Observation. Puis les responsables des deux principales nouvelles Unités de Recherche candidates à intégrer l'OSUG, le LECA et le LEGI, ont présenté leur vision de cette association. Un entretien en tête-à-tête entre le Comité d'AERES et les personnels des Services directement rattachés au directeur de l'OSUG a permis de faire un point sur l'ensemble de ces services. La réunion a été très utilement complétée par des visites de plusieurs de ces services : le service de calcul intensif, les collections géologiques et enfin la ligne FAME de l'ESRF. Enfin, une rencontre avec les représentants de l'ensemble des tutelles (dont l'INSU/CNRS par visioconférence) a permis de recueillir les appréciations de ces tutelles sur les résultats de l'activité de l'OSUG et sa place dans la politique de chaque tutelle.

A l'issue de l'ensemble de ces présentations et visites, le Comité a pu se réunir à huis clos pour élaborer ses principales recommandations et rédiger un pré-rapport.

\section{- Historique de la structure, localisation géographique des chercheurs et description synthétique de son domaine d'activité :}

L'OSUG est l'un des principaux Observatoires des Sciences de l'Univers établis au sein des Universités, sous la forme d'Ecoles Internes, pour y fédérer la politique scientifique dans le domaine des Sciences de l'Univers sous l'égide de l'INSU-CNRS.Il est rattaché à l'Université Joseph Fourier. Ses activités couvrent l'ensemble des domaines des SdU (astronomie-astrophysique, planétologie, sciences de l'environnement, sciences de la Terre solide) ce qui lui donne, sans doute avec l'OSU de Toulouse (OMP) une place exemplaire dans le dispositif national et universitaire des SdU. La quasi-totalité des effectifs de l'OSUG et de ses Unités de Recherche sont implantés sur le campus universitaire Grenoblois.

\section{- Equipe de Direction :}

L'OSUG est dirigé par une équipe composée du directeur Henri-Claude Nataf assisté de deux directeursadjoints chargés de l'enseignement (Philippe Belleudy) et des relations extérieures (Michel Esteves). Un responsable administratif gère les moyens universitaires de la composante.

- Effectifs propres à la structure (personnels affectés spécifiquement à la structure fédérative à la date du dépôt du dossier à l'AERES):

12 personnels techniques et administratifs sont affectés directement à l'OSUG. 


\title{
- Appréciation sur la structure fédérative
}

\author{
- Avis global :
}

L'Observatoire des Sciences de l'Univers de Grenoble (OSUG) est un OSU exemplaire, qui joue pleinement son rôle d'animation et de structuration du champ thématique "Sciences de l'Univers » sur le campus grenoblois et à l'échelle régionale. Sa politique de mise en commun de services techniques et administratifs, conduite avec intelligence et modération, est un vrai succès, de même que le développement des Services d'Observation en son sein. Il a démontré dans ses actions récentes qu'il est le socle sur lequel la structuration de ce domaine peut être entreprise avec efficacité, avec le regroupement de plusieurs Unités de Recherche, en Sciences de la Terre d'un côté, en astronomie-planétologie de l'autre, en grandes UMRs. Il est reconnu par l'ensemble de ses tutelles comme un acteur majeur capable de conjuguer efficacement leurs politiques scientifiques, et possède pour cette raison toute la légitimité nécessaire pour entreprendre dans son prochain mandat un regroupement d'unités plus large, qui enrichira le dispositif de recherche SDU par l'accueil de nouvelles équipes qui l'ouvriront à des domaines complémentaires (écologie) et à des disciplines fondamentales offrant des interfaces fécondes avec les SDU : Physique, mécanique, SHS. Le comité donne un avis très favorable à cette nouvelle extension du périmètre de l'OSUG.

\section{- Points forts et opportunités :}

Les points forts de l'OSUG sont le succès de sa politique de Services d'Observation, et celui de sa politique scientifique au sens large qui est bien reconnue par ses tutelles comme la conjugaison à l'échelle locale de leurs politiques d'Etablissement. L'UMS, malgré sa taille modeste et l'absence de redondances entre les fonctions exercées par ses personnels, est un succès dont il faut féliciter personnels et direction. Les services d'observation, déployés sur un domaine d'action très large, sont également un succès à ce jour. Deux opérations lourdes de regroupement d'Unités en deux UMRs, l'une en astronomie-planétologie, l'autre en Sciences de la Terre, ont été conduites à leur conclusion sous l'égide de l'OSU. Il faudra assurer le succès de ces nouvelles Unités au cours du prochain quadriennal. Enfin le contexte de la structuration scientifique de l'UJF en pôles scientifiques forts, et l'intégration de l'OSUG au pôle MIEU, représente une opportunité d'enrichissement des dialogues interdisciplinaires si l'OSUG mène en parallèle un travail de renforcement de sa politique scientifique et de mise en commun d'actions et de moyens entre ses Unités.

\section{- Points faibles et risques :}

Les effectifs de l'UMS sont sans doute sous-critiques par rapport à l'ensemble des fonctions assurées, et n'offrent pas actuellement de redondance. Cette situation n'a pas empêché un fonctionnement satisfaisant en raison de la motivation et de la compétence des personnels et de l'action appréciée de l'équipe de direction. Face à une nouvelle extension du périmètre de l'OSUG, le comité recommande cependant un développement raisonnable de la taille de l'UMS, qui devra être construit en partie sur une plus grande mise en commun de certaines fonctions entre les Unités, en partie par un effort particulier d'apport des tutelles en Ressources Humaines, parfaitement justifié par le rapport ITA/chercheurs particulièrement et anormalement faible de l'OSUG pris dans son ensemble.

Face au grand nombre de Services d'observations, il faut définir des priorités sur les actions les plus fédératrices, telles que le projet de Centre de Données, qui reçoit notre soutien.

Les relations scientifiques entre la composante astronomie et les sciences de la planète au sens large sont sans doute encore en dessous de leur potentiel de développement, ce malgré la bonne volonté de tous. Au moment où un nouvel élargissement de l'OSUG est programmé, cette préoccupation doit être traitée avec vigueur, à travers des actions et animations sur des thématiques d'interfaces et des aspects « méthodes ». La mise en place d'un véritable Conseil Scientifique, qui manque aujourd'hui, irait dans le bon sens.

\section{- Recommandations :}

Le comité recommande sans réserve et avec vigueur le renouvellement de la structure OSUG pour un prochain quadriennal ; Il félicite l'ensemble des personnels relevant de l'OSUG, au sein de l'UMS et de ses Unités, ainsi que la direction, pour le succès indéniable de l'OSUG dans sa mission, et leur souhaite plein succès pour la nouvelle étape qui s'annonce, avec la construction d'un OSUG plus fort et au périmètre élargi. 
Pour faire face aux défis de cette nouvelle étape, le comité recommande que l'OSUG franchisse une nouvelle étape dans la mutualisation de moyens techniques et administratifs au service de l'ensemble de ses Unités, et avec leur concours. Il soutient le développement de projets de nouveaux services ciblés, tels que le projet de Centre de Données. Le comité souhaite également que l'OSUG se dote d'un Conseil Scientifique capable d'intégrer les dimensions formation, recherche, services communs et services d'observation de ses activités en une politique scientifique forte capable de renforcer la cohésion et l'impact scientifique de l'OSU au moment de son intégration au sein de la composante MIEU de l'Université Joseph Fourier.

\section{- Appréciations détaillées}

\section{- Bilan de l’activité scientifique issue de la synergie fédérative :}

Le travail d'animation scientifique de l'OSUG a des effets concrets en termes de structuration pérenne du champ thématique de l'OSUG. En particulier cela a permis de donner un cadre scientifique motivant à la réalisation des deux fusions de laboratoires «lourdes» dans les domaines Sciences de la Terre et astro-planéto. Le développement des services d'observation, en particulier à travers l'approche des ateliers, est un autre résultat concret de l'activité scientifique à l'échelle de l'OSUG.

Il manque cependant dans les documents présentés un bilan quantitatif des publications communes issues des synergies internes à l'OSU.

\section{- Réalité et qualité de l'animation scientifique :}

L'OSUG joue bien son rôle d'animation scientifique d'un domaine scientifique large, assurant des liaisons entre des Unités plus positionnées sur leur «cœur de métier». Sa démarche de mise en place d'axes thématiques transverses est un succès. Un bon exemple d'animation scientifique commune est offert par le domaine de la géochimie et de l'astrochimie.

L'OSUG pourrait cependant aller plus loin sur d'autre thématiques, et c'est important pour consolider le rassemblement des disciplines de l'OSUG de l'astrophysique aux Sciences de la Planète et aux différents aspects de l'environnement et du développement durable. Les liens entre astrophysique et sciences de la planète restent ténus ; la planétologie par exemple, ou le magnétisme, pourraient être mieux mis à profit pour les développer.

Des synergies intéressantes ont été exploitées dans le domaine du développement des outils (calcul, modélisation), et doivent être approfondies (centre de données, technologies de l'instrumentation...).

\section{- Rôle de l'OSUG vis-à-vis des Services d'Observation :}

L'OSUG et ses Unités de recherche gèrent un ensemble large de Services d'Observation dont beaucoup sont de grande qualité, et jouent un rôle important aux niveaux national et/ou international dans chacune des grandes disciplines de l'OSUG.

En astronomie, l'OSUG joue un rôle moteur dans le développement de SPHERE, instrument de seconde génération du VLT destiné à la détection et la caractérisation des exoplanètes. De même, il est responsable de la Phase A de l'instrument EPICS destiné à poursuivre des objectifs similaires sur le futur E-ELT. L'OSUG contribue également au développement d'instruments de seconde génération pour le VLTI (AMBER, PIONIER, VSI et GRAVITY), à la définition du spectro-polarimètre SPIROU pour le CFHT, à l'étalonnage et à la réduction des données de la mission PLANCK. Enfin, il assure la coordination nationale du JMMC, centre spécialisé dans le développement et la maintenance de logiciels de traitement du signal en interférométrie. Ces Services d'Observation (labellisés AA-SO2 et AA-SO5 pour le JMMC) mettent à profit l'expertise unique des chercheurs du LAOG en matière de spectro-polarimétrie à haut contraste, d'interférométrie spatiale infra-rouge, d'optique intégrée et de traitement du signal. 
A l'interface entre astronomie et planétologie, l'OSUG a amorcé la mise en place de la base de données GHOSST (en cours le labellisation AA-SO5) pour la spectroscopie et la thermodynamique des composés en phase solide. Il s'agit là aussi d'un domaine d'expertise reconnue pour des chercheurs du LPG. Il est à noter que le recrutement à court terme d'un jeune chercheur est vital pour prendre en charge la validation scientifique des données alimentant la base.

Dans le domaine de la physique ionosphérique, l'OSUG gère la participation française au réseau international EISCAT (labellisé AA-SO6) pour l'étude de l'ionosphère par sondage radar à diffusion incohérente.

Dans le domaine de l'hydrologie, les deux services d'observations de l'OSUG, AMMA-Catch en Afrique, OHMCV dans les Cévennes sont gérés par le LTHE. Ce laboratoire avec le soutien de l'OSUG a démontré sa capacité à gérer des SO de cette ampleur, tant pour le maintien des observations que pour l'animation des recherches autour de ces moyens. Ces deux services ont un fort potentiel d'ouverture vers l'extérieur du monde de la recherche dont le LTHE a su tirer tous les avantages.

Le service Glacioclim cogéré par le LTHE et le LGGE est lui aussi une réussite de l'OSUG. L'étude des glaciers y a connu une forte dynamique et a été particulièrement bien valorisée. Le projet d'extension de ce service à l'Himalaya a fait l'objet de recommandations spécifiques par les comités de visite des deux laboratoires concernés.

Enfin, dans le domaine des Sciences de la Terre, l'OSUG est une, si ce n'est la, structure indispensable pour faire fonctionner le service national de sismologie. Il contribue significativement à plusieurs composantes (réseau sismologique SISMALP, réseau large-bande permanent, réseau accélérométrique, GPS, instabilités de versant) en assumant la responsabilité pour la communauté nationale de tâches de service fondamentales.

Les Services d'observation sont donc en général gérés par les Unités de Recherche compétentes, cependant l'OSUG offre le cadre général dans lequel est définie la politique des SO, et encourage le développement de nouveaux Services. De ce point de vue la démarche des ateliers d'observation est très efficace et pertinente pour développer et faire émerger des nouveaux services, ainsi que pour tester leur viabilité à moyen terme.

Le retour sur investissement des moyens mis par l'OSUG dans les Services d'Observation est très bon, tout particulièrement en regard de la situation très tendue des effectifs ITA sur l'ensemble de l'OSUG. Toute extension de ces services devra être précédée d'une analyse précise des moyens complémentaires nécessaires, tout particulièrement en ressources humaines.

Le comité soutient fortement le projet de Centre de données, qui est très fédérateur et très pertinent par rapport à l'évolution des besoins de la communauté : services à valeur ajoutée, data mining, mise en relation des données acquises par l'OSUG et de données d'autres sources. La réalisation de ce projet passe par l'élaboration au niveau de l'OSUG d'un plan de développement précis, qui doit mobiliser toutes les compétences nécessaires, y compris à l'extérieur de l'OSUG. C'est à l'issue de cette phase qu'une expression de besoins doit etre produite et validée, et soumise aux tutelles.

\section{- Pertinence et qualité des services techniques communs :}

D'une manière générale, le comité émet une appréciation très positive sur les activités conduites au sein des services communs de l'OSUG, qui ont été bien mises en lumière par les visites comme par la rencontre avec les personnels de l'UMS. Ci-dessous on trouvera quelques remarques particulières sans prétention à être exhaustifs, ainsi que quelques recommandations générales importantes.

- Calcul intensif : son activité est pertinente et de qualité, conduite en synergie étroite avec les services correspondants de l'UJF.

- Service informatique : il offre un travail de qualité apprécié de tous. On pourrait sans doute aller plus loin dans la mutualisation des activités avec les Unités. Une réflexion particulière devrait être entreprise dans cette direction.

- Communication : cette activité, très importante pour l'OSUG et le domaine thématique qu'il sert, est à soutenir et développer.

- Documentation : la compétence du personnel est reconnue très largement, et les résultats obtenus sont très positifs. Le comité salue en particulier le succès de la mise en place du portail d'archives ouvertes HAL.

Les collections géologiques de Grenoble font partie du patrimoine national et doivent être préservées. L'action de l'OSUG, essentielle dans ce domaine, doit être poursuivie. 
La ligne de lumière FAME de l'ESRF donne à l'OSUG une vraie responsabité nationale, que la direction de l'OSUG assume très bien. Les personnels concernés apprécient leur positionnement à l'interface de la communauté ESRF, de l'OSUG et des utilisateurs de la ligne FAME.

Services administratifs : le développement du portail « composante » est une avancée, le service est apprécié. L'évolution vers une intégration à la future nouvelle composante MIEU de l'UJF doit se faire sans dégrader les services rendus aujourd'hui aux personnels et aux Unités.

Globalement, ces services regroupés dans l'UMS sont performants, les personnels ont su faire reconnaitre leur compétence, et disent apprécier leur cadre de travail. L'action de la direction de l'UMS est appréciée.

Mais ces services sont généralement sous-critiques (pas de redondance). Au moment où l'OSUG envisage d'accroitre son périmètre, il est essentiel que soit conduite une réflexion sur les services à mutualiser au niveau de l'OSUG, sur leurs effectifs, et éventuellement sur les priorités à établir. Un élargissement raisonnable des effectifs de l'UMS, auquel à la fois les tutelles et les Unités doivent participer activement, doit accompagner la dynamique de croissance.

\section{- Role en matière de coordination des politiques des organismes :}

L'OSUG joue pleinement le rôle d'intégrateur de leurs politiques scientifiques que ses tutelles attendent de lui, sur les périmêtres relevant de ses compétences (local et régional) tout en contribuant largement à la visibilité nationale et internationale forte des recherches en Sciences de l'Univers du campus. C'est un succès important et indéniable.

\section{- Role régional :}

L'OSUG joue un rôle majeur dans la structuration scientifique régionale (tutelles, plan campus,...). Il pourrait jouer un rôle encore plus important et visible dans le domaine « environnement et développement durable, par exemple dans le contexte Envirhonalp, en coordination avec ses Unités de recherche.

\section{- Réalité et degré de mutualisation des moyens des unités :}

Il reste limité. On doit pouvoir aller plus loin autour d'un projet de développement précis, accompagnement indispensable de la dynamique d'accroissement du périmêtre scientifique de l'OSUG.

Dans le domaine de l'environnement, préciser les rôles spécifiques à faire jouer à l'OSUG par rapport à une structure telle qu'Envirhonalp. L'OSUG devrait jouer un rôle plus important dans la mise en œuvre des projets et la gestion des plateformes issues des actions d'Envirhonalp, au-delà de la participation des Unités.

\section{- Diffusion de la culture scientifique :}

L'OSUG a pris beaucoup d'initiatives originales et intéressantes dans ce domaine, à la fois vis-à-vis du public et de la formation permanente, telles que le projet d' « opéra de l'Univers ». C'est un vrai succès, il faut encourager les acteurs de ces initiatives à poursuivre et amplifier.

- Pertinence du projet de stratégie scientifique, complémentarité / insertion par rapport aux autres structures fédératives présentes sur ce site :

\section{Avis sur l'accueil de nouvelles unités:}

\section{LECA}

La thématique Ecologie manque au dispositif de l'OSUG aujourd'hui, pour jouer pleinement un rôle dans les enjeux « environnement ». 
L'association (rattachement secondaire) du LECA, qui apporte une Unité active également sur le territoire Alpin, sujet transversal déjà établi au niveau de l'OSUG, est particulièrement pertinente. L'OSUG pourrait bénéficier des implantations du LECA du cadre de la «zone atelier Alpes ». Cette association doit être pleinement reconnue par les tutelles du LECA (INEE...).

\section{LEGI}

Pour mémoire, le MEOM (océanographie) est déjà rattaché à l'OSUG.

L'intégration de la composante « fluides géophysiques » est pertinente et sera un apport intéressant à l'OSUG. L'intégration de l'ensemble du LEGI implique que le LEGI, avec le soutien de ses tutelles s'investisse effectivement dans les thématiques, outils et activités spécifiques de l'OSUG (services d'observation, ingéniérie des systèmes de mesure, savoir-faire en modélisation, bases de données...).

\section{GRCC de PACTE (SHS)}

L'apport de cette équipe est très positif sur les enjeux sociétaux de l'environnement. Il importe de poursuivre les collaborations nationales déjà établies pour être prêts pour les prochaines échéances.

\section{LAME de LSP}

L’apport de cette équipe est intéressant et pertinent sur tous les domaines de l'OSUG.

L'atout « Ingéniérie instrumentale ». Le domaine de l'ingéniérie instrumentale est un domaine fort de l'OSUG à travers l'ensemble de ses Unités. L'OSUG doit veiller au maintien - au minimum - et mieux au développement des capacités de réalisation instrumentale de ses Unités dans ses domaines d'excellence.

\section{Gouvernance et comités de l'OSUG :}

L'élargissement de l'OSUG à de nouvelles Unités, qui est encouragé, est un défi scientifique important pour lui, qui sera de plus à situer dans le contexte nouveau de sa participation à la structuration de la recherche à l'UJF en quelques grands pôles scientifiques.

Il est essentiel que l'élargissement de l'OSUG et son intégration au sein de la composante MIEU de l'UJF soit l'occasion pour l'OSUG et l'ensemble de ses Unités de réfléchir à ce qui les unit, ce qu'ils partagent, à l'évolution des services de l'Observatoire dans l'esprit d'un apport équitable de tous au développement des moyens mis en commun, enfin aux thématiques transverses à développer avec les nouveaux arrivants mais aussi entre les Unités déjà présentes.

Dans cette perspective, la mise en place d'un véritable Conseil Scientifique chargé de définir la stratégie scientifique de l'OSUG est essentielle. Ce Conseil serait chargé auprès de la direction de l'OSUG et de ses Unités d'intégrer les dimensions « recherche », «formation », « observation », « services communs " en une vision plus globale de la politique scientifique. Un développement raisonnable des services communs, techniques, administratifs et d'observation, accompagnement nécessaire de l'élargissement, devrait être subordonné à une réflexion de stratégie scientifique à placer dans le cadre du conseil scientifique.

\section{- Impact de la politique RH de l'UJF :}

Le comité note le manque de perspectives de progression de carrière pour les personnels IATOS de la composante, un problème sans doute plus large que celui de l' OSUG.

\section{- Visibilité de l'UJF à travers l'OSUG :}

L'OSUG est vu de l'extérieur comme un des fleurons du dispositif de formation et de recherche de l'UJF, dont l'excellence en Sciences de l'Univers est bien mise en lumière, valorisée et développée par l'action de l'OSUG. La bonne intégration de l'OSUG à l'UJF est indiscutable, et de l'avis du comité fournit un très bon exemple d'un campus où la réussite de l'OSU est aussi celle de son Université de rattachement. Cette situation excellente doit être préservée, en assurant à l'OSUG qu'il conservera sa visibilité et les moyens de sa politique scientifique dans le nouveau cadre de la structuration de l'UJF en pôles. 
Grenoble, le 5 Mai 2010,

\section{AERES}

Monsieur le Président Jean François Dhainaut

Objet : Réponse de l’Université Joseph Fourier Grenoble 1 au Rapport du Comité de Visite Observatoire des Sciences de l'Univers de Grenoble (OSUG) - UMS 832 - Directeur : HC-Nataf

Monsieur le Président, Cher Collègue,

Nous avons examiné le rapport préliminaire d’évaluation mis en ligne sur votre application le 23 Avril 2010 pour :

L'Observatoire des Sciences de l'Univers de Grenoble (OSUG) - UMS 832 - Directeur : HC-Nataf

Au nom de l'établissement, du directeur de l'OSUG et de l'ensemble de ses personnels et de ses partenaires, nous tenons à vous faire part de nos remerciements pour cette évaluation approfondie.

Nous sommes très reconnaissants aux membres du Comité d'évaluation AERES de l'OSUG qui, sous la présidence de Michel BLANC, ont réalisé une analyse parfaitement pertinente de l’OSUG.

Nous enregistrons avec satisfaction l'approbation des choix stratégiques et prenons bonne note des recommandations que l’AERES adresse aux tutelles et à l’OSUG.

Nous vous prions de recevoir, l'expression de nos cordiales salutations.

\section{P/ Le Président de}

l’Université Joseph Fourier Grenoble I

Farid OUABDESSELAM

P/O Le Vice-président

du Conseil Scientifique de

l’Université Joseph Fourier Grenoble I

Laurent DAUDEVILLE



PJ : Courrier mentionnant les erreurs factuelles relevées dans le rapport préliminaire 